\title{
Steiner Traveler: Relay Deployment for Remote Sensing in Heterogeneous Multi-Robot Exploration
}

\author{
Yuanteng Pei and Matt W. Mutka
}

\begin{abstract}
In the multi-robot exploration task of an unknown environment, human operators often need to control the robots remotely and obtain the sensed information by real-time bandwidth-consuming multimedia streams. The task has military and civilian applications, such as reconnaissance, search and rescue missions in earthquake, radioactive, and other dangerous or hostile regions. Due to the nature of such applications, infrastructure networks or pre-deployed relays are often not available to support the stream transmission. To address this issue, we present a novel exploration scheme called Bandwidth-aware Exploration with a Steiner Traveler (BEST). BEST has a heterogeneous robot team with a fixed number of frontier nodes (FNs) to sense the area iteratively. In addition, a relay-deployment node (RDN) tracks the FNs movement and places relays when necessary to support the video/audio streams aggregation to the base station. Therefore, the main problem is to find a minimum path for the relay-deployment robot to travel and the positions to deploy necessary relays to support the stream aggregation in each movement iteration. This problem inherits characteristics of both the Steiner minimum tree and traveling salesman problems. We model the novel problem as the minimum velocity Flow constrained Steiner Traveler problem (FST). Extensive simulations show BEST improves exploration efficiency by $62 \%$ on average compared to the state-of-theart homogeneous robot exploration strategies. BEST also saves cost by using only half the number of robots compared to the counterpart, while still achieving a $24 \%$ exploration time reduction.
\end{abstract}

\section{INTRODUCTION}

Multi-robot exploration of an unknown environment has been studied extensively in mobile robotics. In many applications of multi-robot exploration, the robot's video and audio streams are required to be sent back to the human operator at the base station (BS). For example, in surveillance applications and search and rescue missions in dangerous areas (such as earthquake and nuclear regions), human operators often need the sensed information immediately and sometimes may control the robots remotely. Besides, the use of human operators as "perceptual sensors" to process camera video is a standard practice for both UAVs and ground robotics [1].

In this paper, we propose a heterogeneous exploration model called Bandwidth-aware Exploration with a Steiner Traveler (BEST). In BEST, a relay-deploying node (RDN) "chases" the remaining frontier nodes $(\mathbb{F} \mathbb{N})$ to deploy relays for them (as shown in Fig. 1). This approach is motivated by the following four reasons. First, prior relay deployment is often infeasible when the environment is unknown and relays

Yuanteng Pei and Matt W. Mutka are with the Department of Computer Science and Engineering, Michigan State University, East Lansing, MI 48824, USA \{peiyuant, mutka\} @ cse.msu.edu.

This work was supported in part by NSF grant No. CNS-0721441. have to be placed the same time as the exploration. Second, the $\mathbb{F} \mathbb{N}$ become "worry-free" of the relay deployment with a clear separation of relay and search robots. Hence, it is flexible for the $\mathbb{F} \mathbb{N}$ to focus on exploration and use any existing exploration scheme.

Third, we may markedly save cost and improve efficiency compared to the homogeneous robot approach where all the robots have to be equipped with relay deployment devices or move backwards from frontier areas to serve merely as relays for the streams. Fourth, BEST does not have the drawback of hard limits on exploration range comparing to a homogeneous robot team. When a homogeneous robot team tries to explore farther away and maintain connectivity to the $\mathrm{BS}$, more robots must retreat from frontier areas to serve as relays. With a fixed number of robots, an increasing number of relay robots will finally prevent the $\mathbb{F} \mathbb{N}$ from moving forward. In BEST, however, a constant number of $\mathbb{F} \mathbb{N}$ is not restrained from the increased distance from the BS.

The key problem is to compute a minimum path for the RDN to travel and find a minimum number of positions to deploy necessary relays to support the stream aggregation in each movement iteration. This problem inherits characteristics of both the traveling salesman and Steiner Minimum Tree with Minimum Steiner Points (SMT-MSP) [2] problems. We model the novel problem as the minimum velocity Flow constrained Steiner Traveler problem (FST),

FST is described as follows: Given (1) a fixed number of $\mathbb{F} \mathbb{N}$ to explore an unknown environment in a synchronized iterative (round by round) fashion with a traveling time and a constant velocity, (2) a relay deploying node (RDN) with a relay deploying time $t_{d}$, (3) a transmission constraint that

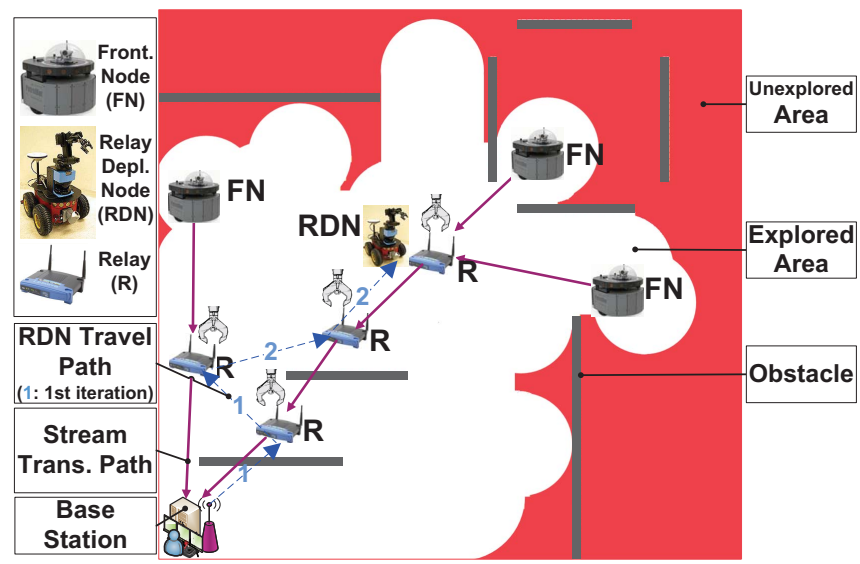

Fig. 1. A relay deploying robot supports the stream aggregation for remote control of frontier nodes in exploration. 
when all $\mathbb{F} \mathbb{N}$ arrive at their destinations in each round, a transmission path formed by relays from each FN to the BS always exists, (This also implies a precedence constraint that needed relays must be deployed by RDN before $\mathbb{F} \mathbb{N}$ arrive), (4) a flow constraint that the number of flows for each relay never exceeds an upper limit $K$. The objective is to find the traveling path for the RDN and positions to deploy relays such that the average traveling velocity for the RDN is minimized.

We minimize the velocity to reduce the energy cost. Because velocity is related to both traveling path length and the traveling time, we need to reduce (1) the number of relays for deployment time to increase the RDN travel time; (2) the traveling path length. Hence, a joint consideration on the number of Steiner points and the traveling paths is needed.

\section{A. Key Contributions}

- To the best of our knowledge, BEST is the first of its kind to (1) jointly consider traveling salesman with flowconstrained Steiner Tree to achieve bandwidth sufficiency and (2) present a heterogenous exploration scheme with relay deployment for unknown environment achieving significant efficiency improvement.

- To solve $\mathrm{R}^{2} \mathrm{BS}$, we formulate the problem as the minimum velocity Flow constrained Steiner Traveler problem (FST). We present an efficient heuristic that takes advantage of existing relays that have unsaturated paths to the $\mathrm{BS}$ to reduce number of relays.

- While designing exploration schemes for an unknown environment, we also wonder what will be the impact of a known global map on performance? Considering the two metrics (1) RDN traveling path length and (2) the number of deployed relays, we find the traveling path length notably benefits from a global map while the number of deployed relays only has marginal improvement.

The rest of the paper proceeds as follows. Related work is summarized in Section II. Section III gives the system model. In Section IV, we formulate the problem and discuss the challenges. Section V presents the BEST scheme. Performance evaluation is in Section VI. Section VII concludes the paper and future work is discussed in the Appendix.

\section{RELATED WORK}

Multi-robot exploration and networked robotics have been extensively studied [3-7]. Maintaining connectivity in mobile robot networks has also attracted increased attention [8].

The connectivity and bandwidth aware exploration (CBAX), is presented in [5,9]. However, it is a homogeneous movement model where robots move backward to serve merely as relays to support connectivity. An interesting work in [10] presents a robot deploying sensor scheme. However, sensors are uniformly deployed without the communication constraints. An online relay deployment to support remote sensing is presented in [11]. However, it assumes a known environment where it does not need to consider the use of existing relays or chasing unpredictable $\mathbb{F} \mathbb{N}$ movements.

\section{SySTEM MODEL}

Robot Model: A robot team includes $|\mathbb{F} \mathbb{N}|$ number of $\mathbb{F} \mathbb{N}$ and a single RDN. Extending BEST with multiple RDNs is discussed in the Appendix. $\mathbb{F} \mathbb{N}$ have traveling, communication, and sensing capability while the RDN can travel, communicate and deploy static relays with a maximum carrying capacity and a deploying time $t_{d}$. We assume BEST satisfies this carrying capacity constraint since it attempts to use a minimum number of relays. Both $\mathbb{F} \mathbb{N}$ and RDN have a communication range $r_{c}$ and a sensing range $r_{s}$ where $r_{c} \geq r_{s} . \mathbb{F}$ move at a constant velocity $v_{f n}$ in all iterations and RDN moves at a changing velocity $v_{r d n}^{t}$ in each iteration $t$. The acceleration and deceleration time is not considered.

Environment Model: A 2D occupancy map is adopted to model the environment as an unknown rectangle region $\mathbb{R}=X \times Y$ with grid cells similar to that in [9]. The cell size equals to the largest robot size. A cell status includes unexplored, exploring, and explored. An explored cell is marked as either free or obstructed. An obstacle cell blocks the traveling path. Such cells cannot be visited by the robots or placed relays. We assume the obstacle distribution in the environment is unknown. A base station is the control center where the human operator remotely monitors and potentially operates the robots when necessary.

Iterative Exploration Model: A fixed number of $\mathbb{F} \mathbb{N}$ explores an unknown environment in a synchronized and iterative (round by round) fashion with a traveling time $T_{f n}^{t}$ in iteration $t$. After all $\mathbb{F} \mathbb{N}$ arrive at their target positions, $\mathbb{F} \mathbb{N}$ and also RDN will receive $\mathbb{F} \mathbb{N}$ 's next iteration target positions and then enter a fixed length sensing and transmitting interval (STI) $T_{s t i}$ to sense the area and mark cells as searched. RDN must finish deploying all necessary relays in round $t$ (denoted as $\mathbb{R}^{t}$ ) before STI starts because the relays are essential to support the transmission. Since the RDN receives $\mathbb{F} \mathbb{N}^{\prime}$ target positions before last round STI, the total time for RDN to travel $T_{r d n}^{t}$ in round $t$ is:

$$
T_{r d n}^{t}=T_{f n}^{t}+T_{s t i}-\left|\mathbb{R}^{t}\right| \cdot T_{d}
$$

We assume $T_{f n}^{t}+T_{s t i}-\left|\mathbb{R}^{t}\right| \cdot T_{d}>0$. We may find that $T_{r d n}^{t}$ is increased with the decreased number of deployed relays. It is especially significant when the deploying time $T_{d}$ is lengthy. All $\mathbb{F N}$ and RDN start traveling from the BS. After the RDN completes the relay deployment in each iteration $t$, it will stop as the last relay deployment position $R_{\text {stop }}^{t}$,

Note that BEST is compatible with any iterative $\mathrm{FN}$ movement strategies for area exploration or other purposes. FN movements can be controlled by a computer program or a human operator at the BS. Under either case, $\mathbb{F} \mathbb{N}$ can only move to known areas because of the unknown obstacles in unknown areas. In BEST, we apply the frontier cell based strategy to maximize sensing gain as in [9].

Wireless Model: The unit-disk model where two nodes $i, j$ can communicate as long as $\operatorname{dist}(i, j) \leq r_{c}$. $\operatorname{dist}(i, j)$ gives the distance from $i$ to $j$. We will consider a probability or a receive signal strength based communication model in future work. Similar to [9], each FN has a uniform flow sending rate, rate send $_{\text {and a link capacity, rate }}$ capacity. A bandwidth 
factor $K$ defines how many flows can a relay maximally carries before exceeding its capacity: $K=\left\lfloor\frac{\text { rate }_{\text {capacity }}}{\text { rate }_{\text {send }}}\right\rfloor$.

In addition, path $(V)$ gives the transmission (routing) path by a vector of nodes $V$, where all adjacent pair of nodes have a distance less than $r_{c}$. We assume the powerful BS have multiple ratios to have sufficient bandwidth for flow aggregation. A similar 15-radio testbed is shown in [12]. The interference between nodes can be resolved by a multichannel dual radio strategy in [13] with proper channel assignment.

\section{Problem Formulation}

With the system model, the formal formulation of the minimum velocity Flow constrained Steiner Traveler problem (FST) is as follows: Given

- a vector of $\mathbb{F} \mathbb{N}^{t}$ positions, a $\mathrm{FN}$ travel time $T_{F N}$, a (partially) explored region $\mathbb{R}^{t}$ with an obstacle-cell subset in each iteration $t=\left\{1, \ldots, n_{i t}\right\}$ with a total number of iterations $n_{i t}$.

- a constant FN velocity $v_{f n}$, FN STI time $T_{s t i}$ and a RDN relay deploying time $t_{d}$,

- a transmission constraint that when all $\mathbb{F N}$ arrive at their destinations in each round, a transmission path formed by relays from each FN to the BS always exists, (This also implies a precedence constraint such that needed relays must be deployed by RDN before $\mathbb{F} \mathbb{N}$ arrive),

- a flow constraint that the number of flows for each relay never exceeds a bandwidth factor $K$,

- placement constraint: no relay is placed on obstacles;

To find (in each iteration $t$ )

- new relay positions $\mathbb{R}^{t}$ (or its subset) in each iteration $t$, along with (a subset of) existing relays $\mathbb{R}^{1 . . n-1}$, to form a directed Steiner tree to connect $\mathbb{F} \mathbb{N}$ to the BS,

- a RDN Hamiltonian path $p a t h_{r d n}^{t}$ from last round stop $\operatorname{position}\left(R_{\text {stop }}^{t-1}\right)$ to travel each position in $\mathbb{R}^{t}$ exactly once, such that the average RDN travel velocity $\overline{v_{r d n}}$ is minimized. $\overline{v_{r d n}}$ depends on both the travel path length and travel time: $\overline{v_{r d n}}=\frac{\sum_{t=1}^{n_{i t}} p a t h_{r d n}^{t}}{\sum_{t=1}^{n_{i t}} T_{r d n}^{t}}$. Mathematically, FST is

$$
\begin{gathered}
\text { Minimize } \overline{v_{r d n}}, \\
\text { s.t. } \exists \operatorname{path}(V), V=\left(i, v_{1}, \ldots, v_{n}, \mathrm{BS}\right), \forall i \in \mathbb{F} \mathbb{N}, v_{i} \in \mathbb{R}^{1 . . t}, \\
\operatorname{dist}\left(v_{j}, v_{j+1}\right) \leq r_{c}, \forall v_{j} \in V, j \leq n, \\
\sum_{i \in \mathbb{F} \cup \mathbb{R}^{1 . . t}}\left|f_{\overrightarrow{i j}}\right| \leq K, \forall j \in \mathbb{R}^{1 . . t}, \\
\operatorname{position}(i) \neq \text { obstacle, } \forall i \in \mathbb{R}^{1 . . t}, \\
\forall t=1, \ldots, n_{i t}
\end{gathered}
$$

Eq. (3) and (4) show there is a valid routing path formed by existing and newly deployed relays from each FN to the BS via deployed relays under the communication range. Eq. (5) gives the bandwidth constraint.

In each iteration $t$, the computation of the Hamiltonian path for RDN $p a t h_{r d n}^{t}$ is assisted by the conversion from asymmetric traveling salesman problem (TSP) similar to that in [11]. Then, we apply the integer linear programming (ILP) formulation for TSP in [14] as below:

$$
\begin{aligned}
\text { Minimize } \sum_{i=1}^{n} \sum_{j=1, j \neq i}^{n} c_{i j} x_{i j}, & \\
\text { Subject to } \sum_{i=1, i \neq j}^{n} x_{i j} & =1, \quad \forall j=1, \ldots, n, \\
\sum_{j=1, j \neq i}^{n} x_{i j} & =1, \quad \forall i=1, \ldots, n, \\
y_{i j} & \geq x_{i j}, \quad \forall i, j=2, \ldots, n, \quad i \neq j, \\
y_{i j}+y_{j i} & =1, \quad \forall i, j=2, \ldots, n, \quad i \neq j, \\
y_{i j}+y_{j k}+y_{k i} & \leq 2, \quad \forall i, j, k=2, \ldots, n, \quad i \neq j \neq k, \\
y_{i j} & =0, \quad \forall i, j=2, \ldots, n
\end{aligned}
$$

Where $c_{i j}$ is the obstacle-aware path length computed by $\mathrm{A}^{*}$ search from position $i$ to $j . x_{i j}$ and $y_{i j}$ are binary and

\begin{tabular}{|c|c|}
\hline Terms & Definitions \\
\hline $\begin{array}{l}\text { FN, RDN, R, } \\
\text { BS, } \mathbb{F N}, \mathbb{R}\end{array}$ & $\begin{array}{l}\text { Frontier nodes, relay-deploying node, deployed relays, } \\
\text { and base station. } \mathbb{F} \mathbb{N}, \mathbb{R} \text { represent } \mathrm{FN} \text { and relay set. } \\
|\mathbb{F N}| \text { gives the number of } \mathbb{F N} \text {. }\end{array}$ \\
\hline $\begin{array}{l}\mathbb{R}^{1 \ldots t-1}, \mathbb{R}^{t} \\
\mathbb{R}^{1 \ldots t}\end{array}$ & $\begin{array}{l}\text { Existing relays deployed before iteration } t \text {, relays de- } \\
\text { ployed in iteration } t \text {, and their union. }\end{array}$ \\
\hline$r_{c}, r_{s}$ & Communication range and sensing range. \\
\hline$T_{r d n}^{t}, T_{f n}^{t}$ & The RDN and FN travel time in iteration $t$ \\
\hline$T_{d}, T_{s t i}$ & $\begin{array}{l}\text { A constant relay deploying time for RDN; A sensing } \\
\text { and transmitting interval for } \mathbb{F N} \text {. }\end{array}$ \\
\hline path $_{r d n}^{t}$ & Traveling path by RDN in iteration $t$. \\
\hline$\frac{v_{f n},}{v_{r d n}}, \quad v_{r d n}^{t}$ & $\begin{array}{l}\text { A uniform FN travel velocity, a RDN travel velocity in } \\
\text { iteration } t \text {, and RDN's average velocity. }\end{array}$ \\
\hline$n_{i t}$ & $\begin{array}{l}\text { Total number of iterations in the iterative movement } \\
\text { model. }\end{array}$ \\
\hline$K$ & $\begin{array}{l}\text { Bandwidth ratio: maximum number of allowed carried } \\
\text { flows per relay. }\end{array}$ \\
\hline$f_{\overrightarrow{i j}},\left|f_{\overrightarrow{i j}}\right|$ & $\begin{array}{l}\text { The flows on edge from } i \text { to } j \text { with the sending rate } \\
\text { rate }\end{array}$ \\
\hline $\operatorname{dist}(i, j)$ & The distance from $i$ to $j$. \\
\hline $\operatorname{path}(V)$ & A transmission path by a vector of nodes $V$. \\
\hline $\mathrm{BR}, \mathbb{B R N}$ & The border relay and its set. \\
\hline
\end{tabular}
$x_{i j}=1$ shows position $i$ is before $j$ immediately in the tour. It is reasonably fast to solve TSP by a branch and cut method with the ILP formulation. Instances with 200 nodes is computed optimally in a couple of minutes [15].

Note that we depict FST with the robotic notations to illustrate our application. A general theoretical description of FST is made possible with the following term changes: (1) $\mathbb{F} \mathbb{N}$ and BS become the terminal points where $\mathbb{F} \mathbb{N}$ are the sending terminals and BS is the base terminal. (2) Existing and current iteration relays become existing and new Steiner points respectively. (3) $R_{\text {stop }}^{t-1} \cup \mathbb{R}^{t}$ becomes the set of cities where $R_{\text {stop }}^{t-1}$ is the starting city for the traveling salesman.

\section{A. Challenges}

There are two major challenges to solve FST. First, FST combines two NP-hard problems: Steiner minimum tree and traveling salesman. The traveling path generation depends on the locations and the number of relays deployed. There is also a trade-off of the relay number and the traveling path length. To minimize the RDN velocity, both the relay number 
and the traveling path need to be reduced. However, it is possible that a relay placement with a least relay number but improper positions will lead to a longer travel path. Besides, the importance of the two factors may vary with different parameter settings of the traveling time $T_{f n}^{t}$ and deploying time $T_{d}$ (recall Eq. 1).

Second, the possible use of existing Steiner points increases the problem complexity. Many related work models the minimum relay placement by SMT-MSP $[2,9,16]$, Compared to SMT-MSP, a main distinction is that when placing relays (new Steiner points) in each iteration $t$, existing relays may be used. It brings a new problem of whether and how to use them.

\section{BEST: BANDWIDTH-AWARE EXPLORATION WITH A STEINER TRAVELER}

We first present BEST's simpler version when the bandwidth ratio $K$ is sufficient large, then we show how we deal with the extra constraint of bandwidth. Before presenting the solution, we first give the definitions.

Definition 5.1: A flow aggregation point is the base terminal to which sending terminals send flows.

Definition 5.2: A border relay $B R_{i}^{1 . . t-1}$ for a $\mathrm{FN} i \in \mathbb{F}$ is an existing relay with the shortest distance to $i$ compared to other existing relays. A BR is qualified to be an aggregation point if there is an unsaturated path from the BR to the BS.

\section{A. BEST with Sufficient Bandwidth}

As discussed in Sec. IV-A, the major difference of FST from SMT-MSP is the availability of existing Steiner points. Now that effectively using $\mathbb{R}^{1 . . t-1}$ in the Steiner tree does not add to the "cost", we design our strategy as follows.

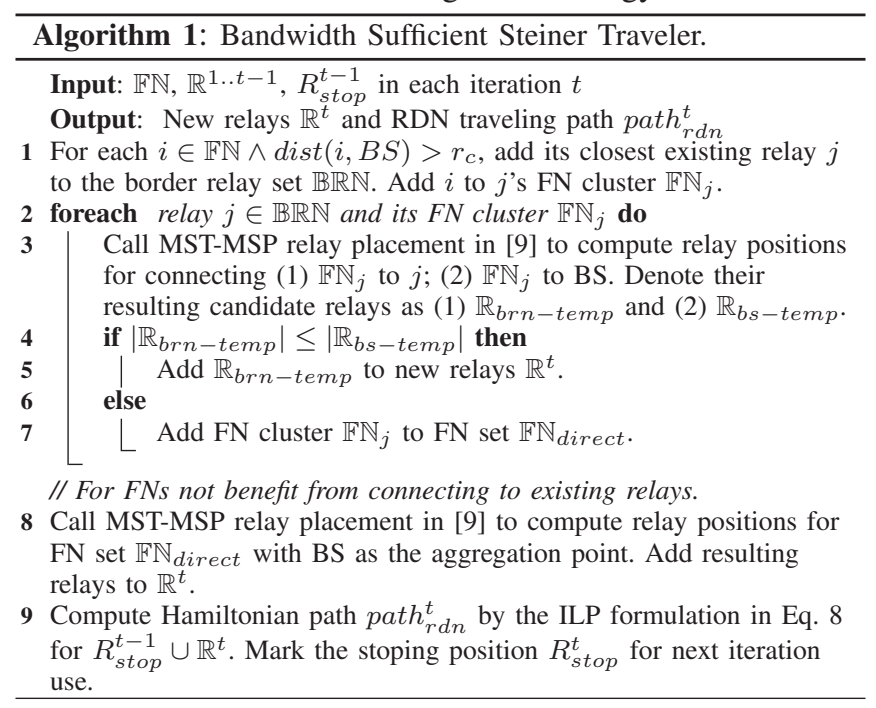

Basic Idea: We cluster $\mathbb{F N}$ to nearby qualified border relay. For each FN cluster, we compare the relay number needed to reach its two potential aggregation points, the border relay and the BS. The border relay is only used when expected to reduce relays. We compute the traveling path afterwards. With $\mathbb{R}^{t}$ and $p a t h_{r d n}^{t}$ for each iteration $t$, the RDN average velocity is then computed. The algorithm is given in Alg. 1.

We attempt to minimize the deployed relay number and only deploy relay when immediately needed. As a result, there is always a valid path from an existing relay to the BS since it was previously used to send streams. Therefore, all flows sent to the border relays will reach the BS. To consider the impact of relay placement on traveling path, we place relays as far as possible from the existing ones with the room for adjustment: when distance divided by $r_{c}$ yields a fractional number.

\section{B. BEST with Constrained Bandwidth}

Previous work [9] handles bandwidth constrained relay placement without existing relays. It guarantees bandwidth adequacy when directly aggregating flows to the BS or the part from $\mathbb{F} \mathbb{N}$ to the border relays. The only unchecked part for bandwidth sufficiency is from the border relays to the BS. Hence, before selecting border relays, we need to check whether unsaturated paths back to the BS exist. The problem resembles the maximum flow problem with vertex capacities. The differences are: (1) We not necessarily need to obtain the "maximum" flow but simply need to check the existence of an unsaturated path (which is also called an argument path in maximum flow). (2) Because the relays are placed by our algorithm, we are able to keep track of a path from each relay to the BS. This is different from the Edmonds Karp algorithm for maximum-flow to use breadth-first search (BFS) to find the argument path each time.

The major modifications on Alg. 1 to support the constrained bandwidth are as follows.

1. Add structures to save path and flow infomation. We maintain a vector of pathToBS for each relay to save the ordered node list on the path from the relay to the BS. It is updated each time we add new relays.

We first enhance the flow constrained relay placement to save the path from each FN to the aggregation point. When aggregating to border relays, pathToBS from BR is appended to the new relays. Besides, another vector of carriedFNflow is defined for existing relays. It shows those $\mathrm{FN}$ whose flow passes this relay in the current iteration. carriedFNflow is reset and cleared at the end of each iteration.

2. Check unsaturated path. Before selecting an existing relay to be a border relay, we check its qualification by checking whether the path back to the BS is still unsaturated: For each node $i \in$ in its pathToBS, carriedFNflow(i).size() is less than $K$. Unqualified border relays cannot be used as aggregation points. When we run out of qualified BRs, all remaining $\mathbb{F} \mathbb{N}$ will connect to the BS without using existing relays.

3. Update carried flows. After we verify using BR as the aggregation point reduces the relay number, the carriedFNflow on the pathToBS is updatd: For each node $i \in$ pathToBS(BR), the cluster of FNs for this BR is inserted into carriedFNflow(i).

\section{Vi. PERformance Evaluation}

\section{Summary}

- We evaluate the exploration efficiency with varying region sizes, number of robots and obstacle ratios.

- We evaluate the performance difference of BEST (given an unknown environment) vs. the solution where a global 


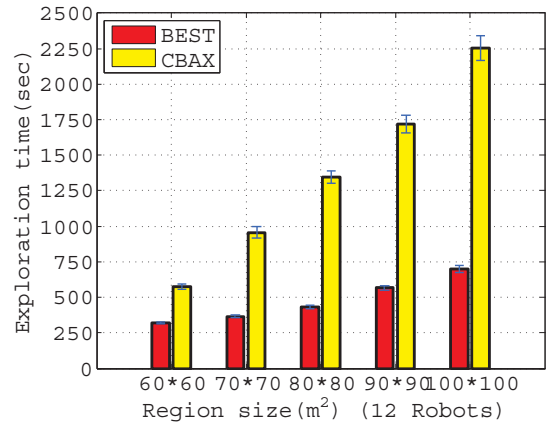

(a) Exploration time in varying region sizes.
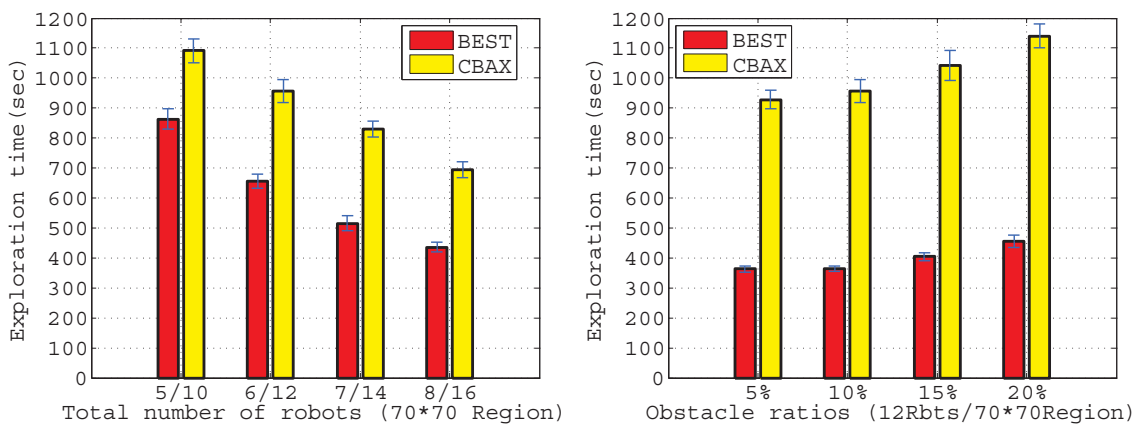

(b) Exploration time in varied number of robots.(c) Exploration time with varying obstacle ratios. (BEST uses half the number of robots of CBAX.)

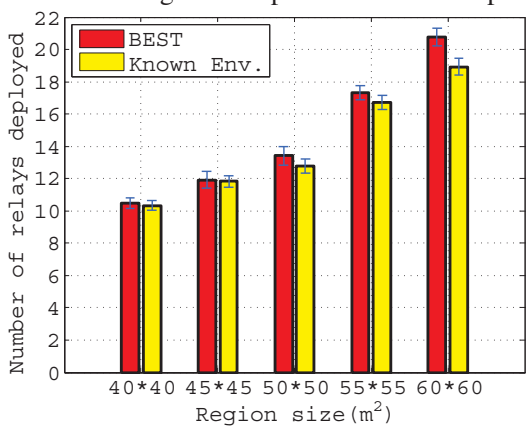

(a) Deployed relay num. in BEST vs. a S-CDS based solution in known environments.

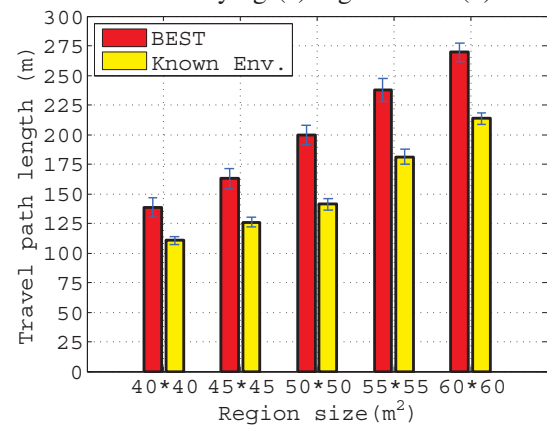

(b) Traveled path length by RDN in BEST

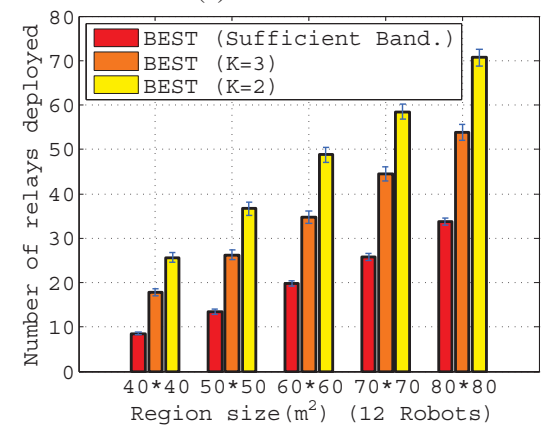

(c) Number of deployed relays with varying bandwidth constraints.

Fig. 3. (a)-(b): Number of deployed relays and traveled path length by RDN in BEST, compared to those results computed with aid of a known environment. (3) Impact of varying bandwidth constraints on the number of deployed relays.

obstacle distribution is known and thus relay positions and traveling paths can be globally optimized.

- Besides, the impact of bandwidth constraints on deployed relay number is investigated regarding different $K$ values.

\section{A. Simulation Setup and Parameters}

We develop a simulation program in $\mathrm{C}++$ for robot exploration. The traveling salesman problem is solved in part by CPLEX V12.2. The unknown exploration region is represented by the grid-map model with $X \times Y$ cells. Each cell has an edge length of 1 meter, and the BS is located at the left-bottom of the region. Obstacles are randomly generated with the ratio (percentage of obstructed cells out of total cells) given by the input. A default bandwidth factor $K$ is set at 3 and obstacle ratio is set at $10 \%$, unless specified otherwise. The communication range $r_{c}$ is $12 \mathrm{~m}$ and the sensing range $r_{s}$ is $6 \mathrm{~m}$. The relay deploying time $t_{d}$ is set as 1 second and FN's $T_{s t i}$ is set as 3 seconds. FN has a constant travel velocity of $0.5 \mathrm{~m} / \mathrm{sec}$. All results are the average obtained by running tests 30 times with different environments of randomly generated obstacles. The error bars show the $95 \%$ confidence interval.

\section{B. Exploration Efficiency}

Among the current exploration strategies with connectivity and bandwidth awareness for an unknown environment, the connectivity and bandwidth aware exploration (CBAX) [5,9] uses less exploration time compared to strategies in [17,18], therefore we choose CBAX as the comparison counterpart on exploration efficiency rather than those in $[17,18]$. CBAX explores the unknown environment iteratively and dynamic selects and places a minimum subset of robots to be the relays in each iteration while the remaining robots to be frontier nodes, given a fixed total number of robots.

The exploration time is computed from the start to the time when $95 \%$ of the region is searched. Fig. 2(a) shows the exploration time with varied region sizes from $60 \mathrm{~m} * 60 \mathrm{~m}$ to $100 \mathrm{~m} * 100 \mathrm{~m}$. BEST reduces the exploration time by $62.0 \%$ on average compared to CBAX given a fixed number of 12 total robots. The exploration time reduction is slightly more notable with larger regions. The reduction increases from $44.1 \%$ in $40 \mathrm{~m} * 40 \mathrm{~m}$ regions to $69.0 \%$ in $100 \mathrm{~m} * 100 \mathrm{~m}$ regions. The major reason is that more robots move backwards to the BS to serve as relays in CBAX, while BEST uses a constant number of robots for exploration.

The average RDN travel velocity in the 5 regions is $1.07 \mathrm{~m} / \mathrm{sec}, 1.14 \mathrm{~m} / \mathrm{sec}, 1.13 \mathrm{~m} / \mathrm{sec}, 1.01 \mathrm{~m} / \mathrm{sec}, 0.95 \mathrm{~m} / \mathrm{sec}$ respectively, approximately 2 times the FN travel velocity. Applying multiple RDNs will relieve the workload of a single RDN and thus decrease the travel velocity. Besides, Fig. 2(b) demonstrates BEST with half the number of robots (e.g., a four-robot team of $1 \mathrm{RDN}+3 \mathrm{FNs}$ in BEST compared to a eight-node team in CBAX) still outperforms CBAX in exploration efficiency. In a $70 \mathrm{~m} * 70 \mathrm{~m}$ region, a half number team in BEST remains $24.1 \%$ faster than CBAX on average.

In addition, we evaluate the impact of different obstacle ratios on the exploration efficiency. Fig. 2(c) shows BEST performances similarly to CBAX with increased ratio of obstacles from $5 \%$ to $20 \%$. There is a climb of $18.6 \%$ for 
CBAX and a rise of $20.4 \%$ for BEST in exploration time.

\section{Comparing to a Solution with Known Environments}

A known environment with obstacle distribution enables optimization of the relay placement and traveling paths under a global view. BEST is designed for exploring an unknown environment. Although only a partial (local) map is exposed for the BS to compute the relay positions and traveling paths in each iteration, it is desirably to know how much we may improve if a global view was provided.

The solution to FST given a known environment is briefly described here. It is a simplified and partial version of the "STARS" solution for a known environment in [11]. Given the obstacle distribution, we compute the sensing positions, relay positions and traveling paths by modeling using set cover, Steiner connected dominating set (S-CDS), and traveling salesman problems (all are NP-hard), respectively.

Fig. 3(a) shows BEST uses only an average of $4 \%$ more relays compared to the S-CDS based solution (both given sufficient bandwidth conditions). It shows that inaccessibility to a global map does not hurt much for deploying relay placement. On the other hand, the extra traveled path length compared to the S-CDS solution is a more notable $30.7 \%$ on average, according to Fig. 3(b). An explanation is that the global map will help to choose the shortest paths globally, leading to significant savings in path length. For the relay placement, however, a local placement, which attempts to"stretch out": sparsely placing relays to be as far as possible to existing ones, performs similar to a global S-CDS to connect the BS to any sensing position, which together covers the whole region.

Fig. 3(c) shows the bandwidth constraint's impact on the number of relays deployed. The number of relays rises $82.8 \%$ for $K=3$ and another $68.9 \%$ for $K=2$ on average respectively compared to the bandwidth sufficient case. The results demonstrate the significant impact of QoS on the number of relays: when no unsaturated path exists, new relays are deployed.

\section{CONCLUSION}

We propose BEST, or Bandwidth-aware Exploration with a Steiner Traveler for an unknown environment. BEST computes the relay positions and traveling paths for the relay-deploying robot to keep track of a group of frontier robots. BEST enables bandwidth-aware real-time multimedia transmissions to support remote sensing and control of a robot team. In addition, we show BEST is extensible with multiple RDNs.

We model the problem by the minimum velocity Flow constrained Steiner Traveler problem (FST). FST combines two of the most important combinatorial optimization problems: the traveling salesman and the Steiner minimum tree problems. Our solution to FST places new relays to connect to existing ones with unsaturated paths rather than to the BS when using existing relays can reduce the number of relays.

BEST significantly improves the exploration efficiency compared to existing homogeneous robot exploration strategies. We also find a marginal improvement in relay number but a notable traveling path length reduction, if a global obstacle distribution was known.

\section{REFERENCES}

[1] M. Lewis, H. Wang, P. Velagapudi, P. Scerri, and K. Sycara, "Using humans as sensors in robotic search," in FUSION 2009.

[2] X. Cheng, D. Du, L. Wang, and B. Xu, "Relay sensor placement in wireless sensor networks," Wireless Networks, vol. 14, no. 3, 2008.

[3] A. Haumann, K. Listmann, and V. Willert, "Discoverage: A new paradigm for multi-robot exploration," in ICRA 2010.

[4] P. Brass, F. Cabrera-Mora, A. Gasparri, and J. Xiao, "Multirobot tree and graph exploration," Robotics, IEEE Transactions on, no. 99, 2011.

[5] Y. Pei, M. Mutka, and N. Xi, "Connectivity and bandwidth aware real-time exploration in mobile robot networks," in Wireless Cоттиnications and Mobile Computing, WCM. (In press, published online).

[6] P. Mukhija, K. Krishna, and V. Krishna, "A two phase recursive tree propagation based multi-robotic exploration framework with fixed base station constraint," in IROS 2010.

[7] H. Liu, A. Nayak, and I. Stojmenovic, "Localized mobility control routing in robotic sensor wireless networks," in MSN 2007.

[8] J. Reich, V. Misra, D. Rubenstein, and G. Zussman, "Connectivity maintenance in mobile wireless networks via constrained mobility," in INFOCOM 2011.

[9] Y. Pei, M. Mutka, and N. Xi, "Coordinated multi-robot real-time exploration with connectivity and bandwidth awareness," in ICRA 2010.

[10] G. Fletcher, X. Li, A. Nayak, and I. Stojmenovic, "Back-tracking based sensor deployment by a robot team," in IEEE SECON 2010.

[11] Y. Pei and M. Mutka, "STARS: Static relays for multi-robot real-time search and monitoring," in DCOSS 2011.

[12] S. Kakumanu and R. Sivakumar, "Glia: a practical solution for effective high datarate wifi-arrays," in MobiCom 2009.

[13] Y. Pei and M. Mutka, "Joint bandwidth-aware relay placement and routing in heterogeneous wireless networks," in ICPADS 2011.

[14] S. C. Sarin, H. D. Sherali, and A. Bhootra, "New tighter polynomial length formulations for the asymmetric traveling salesman problem with and without precedence constraints," Operations Research Letters, vol. 33, no. 1 , pp. $62-70,2005$.

[15] N. Ascheuer, M. Jünger, and G. Reinelt, "A branch \& cut algorithm for the asymmetric traveling salesman problem with precedence constraints," Comput. Optim. Appl., vol. 17, 2000.

[16] D. Du and X. Hu, Steiner Tree Problems In Computer Communication Networks. World Scientific Publishing Co., 2008, pp. 177-193.

[17] M. N. Rooker and A. Birk, "Multi-robot exploration under the constraints of wireless networking," Control Engineering Practice, vol. 15, no. 4, 2007.

[18] A. Franchi, L. Freda, G. Oriolo, and M. Vendittelli, "The sensor-based random graph method for cooperative robot exploration," Mechatronics, IEEE/ASME Transactions on, vol. 14, no. 2, April 2009.

[19] G. Gutin, A. Punnen, A. Barvinok, E. K. Gimadi, and A. I. Serdyukov, "The traveling salesman problem and its variations," 2002.

\section{APPENDIX}

The future work of BEST is briefly described as follows.

A. Extension with Multiple Relay-deploying Robots

Multiple RDNs reduce the work load of each RDN and make the solution more scalable with increase in $|\mathbb{F N}|$ and flow sending rate. Multiple relay deploying robots are a natural consideration when a single RDN has a high workload of relay deployment and a lengthy traveling path to keep track a large number of $\mathbb{F} \mathbb{N}$.

With $n$ number of RDN, we may dynamically partition the $\mathbb{F N}$ to different clusters and assign the RDN to its closest cluster of FNs. The partition's objective may be set to (1) achieve a work load balance for each RDN: minimize the largest difference of $\overline{v_{r d n}}$ among RDNs; (2) reduce the total energy cost: minimize the sum of all $\overline{v_{r d n}}$ among RDNs.

\section{B. Towards Traveling Path Aware Relay Deployment}

Currently BEST solves FST problem by first computing the relay positions. With the relay positions as input, the traveling path is obtained by computing the Hamiltonian path. In a more integrated approach, it is desirable that the relay placement outputs a set of candidate positions where the next step can further evaluate which candidates are better. The uncertainty of the positions for the traveler to travel resembles the Generalized TSP problem [19], where the position set $G$ is partitioned into clusters and the objective is to find the shortest cycle in $G$ which passes at least one position in each cluster. 